Proc. Indian Acad. Sci. (Eart?. Planet. Sci.), Vol. 89, Number 2, July 1980, pp. 239-248. (C) Printed in India.

\title{
Electrical resistivity anomalies over hydrocarbon bearing structures
}

\author{
V K GAUR, SRI NIWAS and N R GARG* \\ Department of Earth Sciences, University of Roorkee, Roorkee 247 672, India \\ * Gujarat Water Resources Development Corporation Limited, Rajkot, India
}

MS received 19 August 1979 ; revised 3 May 1980

\begin{abstract}
The present study is aimed at working out the possible resistivity anomalies associated with hydrocarbon bearing structures. The anomaly due to a typical two-dimensional anticlinal structure filled with hydrocarbon, and overlying a basement of infinite resistivity was computed using the conformal mapping technique. A more realistic and elaborate model, which could not be simplified by conformal mapping, was studied using the finite element method. This model consisted of a two-dimensional anticlinal structure filled with oil or gas-embedded midway in a homogeneous layer which itself overlies a half-space of infinite resistivity, the electrical resistivity of the hydrocarbon bearing structure being simulated as infinite.
\end{abstract}

Keywords. Electrical resistivity; hydrocarban; structures.

\section{Introduction}

Techniques of oil exploration in current use are mostly indirect in the sense that they are designed to locate structures which are most favourable for the occurrence of oil. Confirmation of the presence of oil can be made only after actual drilling which involves a careful balance of cost against the possible return. However, recent developments in the use of direct geophysical techniques for locating hydrocarbons offer possibilities of improving the chances of success. It, therefore seems feasible now to mount vigorous efforts to investigate the role of electrical methods in a petroleum exploration.

Geoelectric methods may under favourable conditions, help detection of oil directly because of its unusually very high resistivity. It seems desirable to compute theoretical or experimental responses of idealised structures containing hydrocarbons. The present analysis is an attempt in this direction.

We have selected the following models as a first step towards evolving more realistic models of hydrocarbon deposits, as more confidence has been gained in developing computer simulation techniques. The essential feature of all these models is a two-dimensional anticlinal form of anomalous resistivity in these cases of infinite resistivity, which simulates oil or gas accumulations. 
Model I consists of buried two-dimensional anticlinal structures filled with oil or gas, overlying a basement of infinite resistivity (figure 1A).

Model II is a variation of the above model in the sense that the anticlinal structure containing hydrocarbons is embedded midway in a homogeneous layer which itself overlies a semi-infinite medium of high resistivity (figure 2).

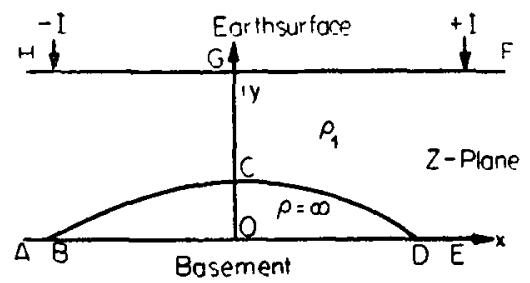

10)

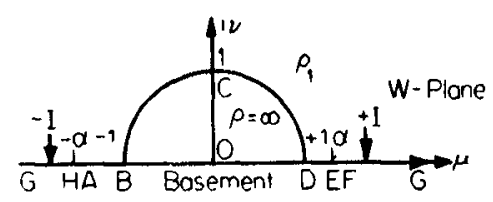

(b)

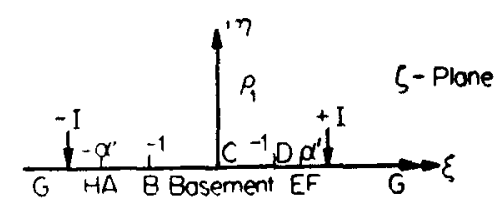

1c)

Figure 1. Transformed geometry of the model I. (a) Geometry of the problem. (b) Conformal transformation of the geometry shown in figure a. (c) Conformal transformation of the geometry shown in figure $\mathbf{c}$.

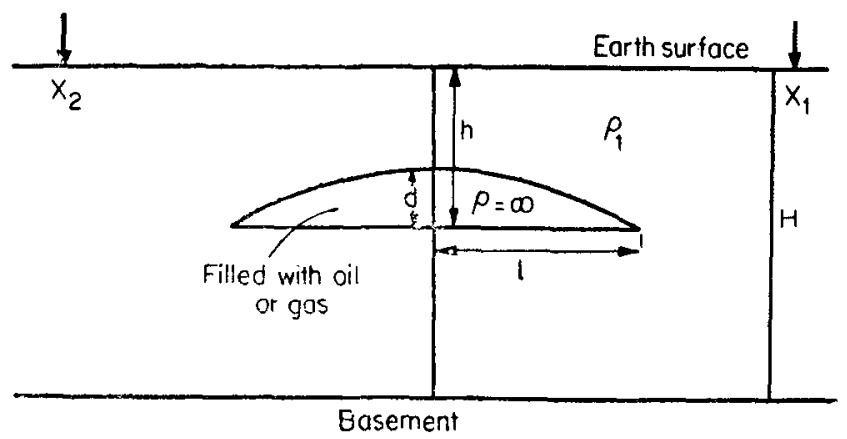

Figure 2. Geometry of the model II. 
A pair of infinitely long line source and sink parallel to the strike of the structure are impressed over the surface of the earth. It may, however, be mentioned here that whilst it is mathematically essential to consider line-source in a theoretical analysis, it will not be necessary to use line source in practice in order to utilise such theoretical results for interpretation of the observed data. It can be shown (Tranter 1956; Roy and Jain 1961; Naidu 1967; Mufti 1976) that measurements made in the field with point source can be transformed to yield corresponding values which would have been obtained if line source had been used. The data so transformed can then be interpreted in the light of theoretical results obtaineds

A closed form solution was obtained for model I using the conformal mapping technique. Computed values of apparent resistivity have been presented it. figures 3 to 5 . Model II was analysed using the finite element method which in essentially a numerical method for modelling complex problems.

\section{Solution of the problems}

\subsection{Model I (figure 1a)-Solution using the conformal mapping technique}

Figure la represents the geometry of the model. ABDO is the line of basem en over which the anticlinal structure BCD filled with hydrocarbon is resting and it is overlain by material of resistivity $\rho_{1}$. Line HF represents air-earth contact, $+I$ is the position of infinite line source and $-I$ is the position of infinite line sink. Two successive transformations are required to reduce this model into one involving two homogeneous half-spaces (figure 1c). Since many geological structures, generally extend for long distances in some direction, they can be approximated to idealised two-dimensional structures. The method of conformal mapping is the most convenient and perhaps the only analytical method available for simplifying the geometry of such complex problems, so that a given curve may be completely mapped on the real axis of the transform plane, the only prerequisite being that the function should be analytic.

The reciprocal transformation in respect-of figures $1 \mathrm{a}$ and $1 \mathrm{~b}$ can be expressed by the relation

$$
\begin{aligned}
z & =\frac{h}{\pi} \ln \frac{a+w}{a-w} \\
z & =x+i y, \\
w & =u+i v, \\
h & =\text { the depth of the base of the structure from the surface of the earth, } \\
2 l & \text { is the length of the base of the anticline, }
\end{aligned}
$$
and $l=\frac{h}{\pi} \ln \frac{a+1}{a-1}$.

The next transformation in respect of figures $1 \mathrm{~b}$ and $\mathrm{lc}$ can then be obtained from the following relation:

$$
\zeta=\frac{1}{2}\left(w+\frac{1}{w}\right),
$$


where $\zeta=\xi+i \eta$.

The solution for the electric potential in the transformed $\zeta$-plane is easily obtained using the conditions (i) that the potential gradient across the real axis vanishes, (ii) that the poteatial tends towards zero on the semicircle with infinite radius in the upper-half-plane and (iii) that the current electrodes are at finite distance.

The harmonic potential $V(\zeta)$ on the surface of the earth $(\eta=0)$ satisfying the above boundary conditions due to a source $\left(\xi_{1}, 0\right)$ and $\operatorname{sink}\left(-\xi_{2}, 0\right)$ is given by

$$
V(\zeta)=\frac{I_{T} \rho_{1}}{\pi}\left[-\ln \left(\xi-\xi_{1}\right)+\ln \left(\xi+\xi_{2}\right)\right]
$$

where $I_{T}$ is the current per unit length flowing through the current electrode and $\rho_{1}$ is the resistivity of the surrounding medium.

Inverting the expression for the electric-potential in the $\zeta$-plane to the corresponding value on to the original z-plane, using transformations (1) and (2), can be written as

$$
\begin{aligned}
& V(z)=\frac{I_{T} \rho_{1}}{\pi} \\
& \times \ln \left[\frac{\left(\frac{\exp \pi x / h+1}{\exp \pi x / h-1} a+\frac{\exp \pi x / h-1}{\exp \pi x / h+1} \frac{1}{a}\right)-\left(\frac{\exp \pi x_{1} / h+1}{\exp \pi x_{1} / h-1} a+\frac{\exp \pi x_{1} / h-11}{\exp \pi x_{1} / h+1 a}\right)}{\left(\frac{\exp \pi x / h+1}{\exp \pi x / h-1} a+\frac{\exp \pi x / h-1}{\exp \pi x / h+1} \frac{1}{a}\right)+\left(\frac{\exp \pi x_{2} / h+1}{\exp \pi x_{2} / h-1} a+\frac{1}{\exp \pi x_{2} / h+1 a}\right)}\right]^{-1}
\end{aligned}
$$

Using the expression for the Schlumberger apparent resistivity given by

$$
\rho_{a}=\frac{\pi}{I_{T}^{-}}\left(\frac{1}{\left(x_{2}+x\right)}+\frac{1}{\left(x_{1}-x\right)}\right)^{-1} \frac{d V(z)}{d x}
$$

One obtains the following expression for the apparent resistivity over the first model (figure 1a).

$$
\begin{aligned}
\rho_{a} / \rho_{1}=2 & \frac{\left(x_{1} / h-x / h\right)\left(x_{2} / h+x / h\right)}{\left(x_{1} / h+x_{2} / h\right)} \\
& \times \exp (\pi x / h)\left\{a-\frac{[\exp (\pi x / h)-1]^{2}}{[\exp (\pi x / h)+1]^{2} a}\right\}\left(A_{1}+A_{2}\right) \\
& \times\left(\frac{1}{\left\{[\exp (\pi x / h)+1] a+\frac{[\exp (\pi x / h)-1]^{2}}{[\exp (\pi x / h)+1] a}\right\}-[\exp (\pi x / h)-1] A_{1}}\right) \\
& \left.\times \frac{1}{\left(\left\{[\exp (\pi x / h)+1] a+\frac{[\exp (\pi x / h)-1]^{2}}{[\exp (\pi x / h)+1] a}\right\}+[\exp (\pi x / h)-1] A_{2}\right.}\right)
\end{aligned}
$$


where $\quad a=\frac{\exp (\pi l / h)+1}{\exp (\pi l / h)-1}$ and $A_{k}=\frac{1}{2}\left(\frac{\exp \left(\pi x_{k} / h\right)+1}{\exp \left(\pi x_{k} / h\right)-1} a+\frac{\exp \left(\pi x_{k} / h\right)-1}{\exp \left(\pi x_{k} / h\right)+1} \frac{1}{a}\right)$.

$$
(k=1,2) \text {. }
$$

When the structure is absent ( $l=0$, two layer case) the expression for $\rho_{a} / \rho_{1}$ is as follows :

$$
\begin{gathered}
\frac{\exp \left[\pi / 2 h\left(x_{1}+x\right)\right] \operatorname{cosec}\left[\frac{\pi}{2 h}\left(x_{1}+x\right)\right]}{\rho_{1}}=\frac{\pi}{2 h} \frac{+\exp [-\pi / 2 h(x-x)] \operatorname{cosec}\left(\frac{\pi}{2 h}\left(x_{1}-x\right)\right)}{\frac{1}{x_{1}+x}+\frac{1}{x_{1}-x}}
\end{gathered}
$$

Expression (7) is the same as expression (97) of Parasnis (1965).

\subsection{Model II}

Since it was not possible to transform the geometry (figure 2) of this model into a simple form, it could only be studied by the finite element method (FEM).

The finite element method is a numerical technique used for obtaining approximate solutions for a wide variety of problems. It is akin to the classical techniques and can be employed in problems involving complex geometrics which may not lend themselves easily to an analytic solution.

The various steps involved in solving the problem by FEM are

(i) discretisation of the solution region,

(ii) selection of interpolation function,

(iii) element properties,

(iv) assembling of the element properties to obtain the system equations, and

(v) solution of the system equations.

\section{Formulation}

The continuity equation for the current density in a two-dimensional cartesian system can be written as follows in terms of the electrical potential $\bar{V}$

$$
\frac{\delta}{\delta x}\left(\sigma x \frac{\delta \bar{V}}{\delta x}\right)+\frac{\delta}{\delta y}\left(\sigma y \frac{\delta \bar{V}}{\delta y}\right)+Q=0
$$

subject to the specified boundary conditions

$$
\bar{V}=\bar{V}_{0}(x, y) \text { on the surface of the system. }
$$

In the present case $\sigma$ is the electrical conductivity and $Q$ the internal current source. In the model, $\bar{V}$ satisfies the Laplace's equation at all points not occupied by electromotances.

The numerical solution of equation (8) can be obtained through a variational approach, i.e., from the energy functional which is then minimised. The required 
energy density function $(J E)$, where $J$ is the current density and $E$ is the electrical field, can be written as

$$
I(\bar{V})=\frac{1}{2} \int_{v}\left[\sigma_{*}(\delta \bar{V} / \delta x)^{2}+\sigma_{y}(\delta \bar{V} / \delta y)^{2}\right] t d x d y
$$

where $t=$ thickness of two-dimensional continuum.

The solution domain has been divided into various quadrilateral elements of $n$ nodes each ( 8 in the present case), the potential within each of which is given by

$$
\bar{V}=\sum_{i=1}^{n} N_{i} V_{i}
$$

where $\bar{V}$ is the potential at any point within the element, $V_{i}$ represents the potential at the $i$ th node and $N_{i}$ is the associated interpolation polynomial shape function (parabolic in the present work).

The minimisation of energy functional in the solution region is achieved by equating its first derivative with respect to the potential at the $i$ th node equal to zero, i.e.

$$
\delta I(\bar{V}) / \delta V_{i}=0 \text { for all } i \text { 's. }
$$

Equation (11) can be written as

$$
\delta I(\bar{V}) / \delta \bar{V}_{i}=\sum_{e}^{n_{e}} \frac{\delta I_{e}(\bar{V})}{\delta \bar{V}_{i}},
$$

where $I_{0}$ is the energy stored within an element. For an element, one can writ]

$$
\delta I_{e} / \delta \bar{V}_{i}=0
$$

or $[S]^{e}\{\bar{V}\}^{\bullet}=0$

where $S_{i j}=\int_{v}\left(\sigma_{z} \frac{\delta N_{i}}{\delta x} \frac{\delta N_{I}}{\delta x}+\sigma_{y} \frac{\delta N_{i}}{\delta y} \frac{\delta N_{i}}{\delta y}\right) t d x d y$

$S]^{e}$ can be written as

$$
\int_{0}[G]^{r}[D][G] t d x d y
$$

where $\quad[D]=\left[\begin{array}{ll}x & 0 \\ 0 & y\end{array}\right]$,

and $\quad[G]=\left[\begin{array}{ccc}\frac{\delta N_{1}}{\delta x} \frac{\delta N_{2}}{\delta X} & \cdots & \frac{\delta N_{n}}{\delta x} \\ \frac{\delta N_{1}}{\delta y} \frac{\delta N_{2}}{\delta y} & \cdots & \frac{\delta N_{n}}{\delta y}\end{array}\right]$.

If the geometry of the element and the shape function are known, the above terms can be evaluated by using the numerical integration techniques. 
The same shape functions which define the unknown potential function in an element are used to map the element in the $x-y$ coordinate system also

$$
x=\Sigma N_{i} x_{i} \text { and } y=\Sigma N_{i} y_{i},
$$

where $x_{i}$ and $y_{i}$ are the coordinates of the nodes in $x-y$ coordinate systems.

If the function [ $\left.N_{i}\right]$ are so chosen that $\bar{V}$ satisfied the continuity and convergence criteria in the $\xi-\eta$ coordinate system, then $[S]^{\mathrm{e}}$ in the cartesian coordinate system can be derived using a few simple transform. Thus all derivative compo. nents transform as

$$
\left\{\begin{array}{l}
\delta N_{i} / \delta_{\bullet} \\
\delta N_{i} / \delta y
\end{array}\right\}=[J]^{-1}\left\{\begin{array}{l}
\delta N_{i} / \delta \xi \\
\delta N_{i} / \delta \eta
\end{array}\right\}
$$

in which $[J]$ is the Jacobian matrix

$$
[J]=\left[\begin{array}{ll}
\delta x / \delta \xi & \delta y / \delta \xi \\
\delta x / \delta \eta & \delta y / \delta \eta
\end{array}\right]
$$

In two dimensions the volume element becomes

$$
d v=t d x d y=t \operatorname{det}[J] d \xi d \eta
$$

The limits for integration of the equation for each element are \pm 1 . With these transformations the matrices are evaluated using numerical integration schemes.

The numerical values of parameters of model II chosen are as follows:

$$
\begin{aligned}
& d / h=0.47, \\
& H=2 h,
\end{aligned}
$$

and $\quad x_{1} / h=x / h=2 \cdot 0$,

where $H$ is the depth of the basement.

\section{Analysis of the numerical results}

Expressions for the apparent resistivity for the above models were calculated using an electronic computer (IBM/370) and these are plotted graphically in figures 3-6 The excitation current is assumed to be introduced in the earth through line electrode parallel to the strike of the structure, for reasons already explained above.

Figure 3 graphically represents the variation of apparent resistivity with the position of measuring potential electrode for fixed current electrode separation $\left(x_{1} / h=x_{2} / h=4 \cdot 0\right)$ and varying $d / h$ values, the $d / h=0$ representing the case when the structure is absent. These curves show that in the absence of the structure, i.e., corresponding to a two-layer case, the apparent resistivity curve possesses a regular hump when the current electrodes are at a finite distance. The $p_{a} / p_{1}$ curves show significant variations in shape as compared with those for $d / h=0$, when the structure is present and the dissimilarity becomes more pronounced with the increasing size of the structure (vide figure 4) attaining a maximum just 


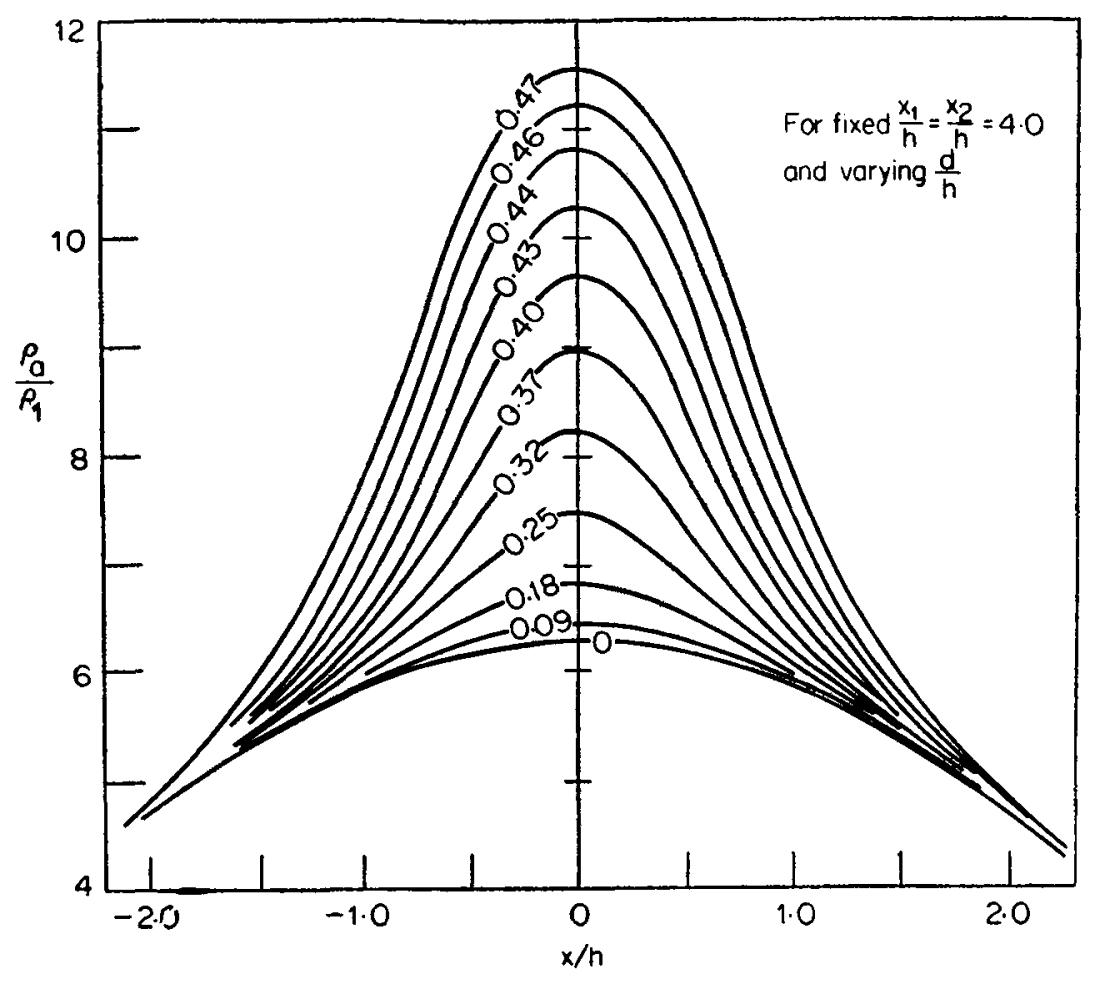

Figure 3. Plot of $\rho_{a} / \rho_{i}$ vs $x / h$ when current electrodes are symmetrically placed about the axis of the structure (conformal mapping technique).

above its axis when the current electrodes are symmetrically disposed. Figure 5 shows the plot of $\rho_{a} / \rho_{1}$ vs $S\left(S=x_{1}+x_{2} / 2 h\right)$ for different values of $d / h$, at a point lying over the axis of the structure. It can be observed that whilst the slope of the $\rho_{a} / \rho_{1}$ curve is about $57^{\circ}$ (for $d / h=0$ and $S$ greater than about 2) as expected over a two-layered earth with a resistive substratum, it exceeds this value when the structure is present, increasing with its size, thereby providing a clue to the presence of the structure as well as a means of calculating its dimensions.

Figure 6 shows the same variation for model II using the finite element method. The nature of the curve is broadly similar to that obtained for model I described above.

\section{Discussion and conclusion}

The primary aim of the exercise was to investigate the pattern of resistivity anomalies gleaned through various electrode configuration over an anticlinal enclosure filled with oil or gas and buried in a homogeneous half-space (with the basement lying at a considerable depth). However, it was not possible to solve this problem by conventional mathematical techniques. A slightly modified model was therefore adopted in which the basement was shifted from infinity to the lower plane 


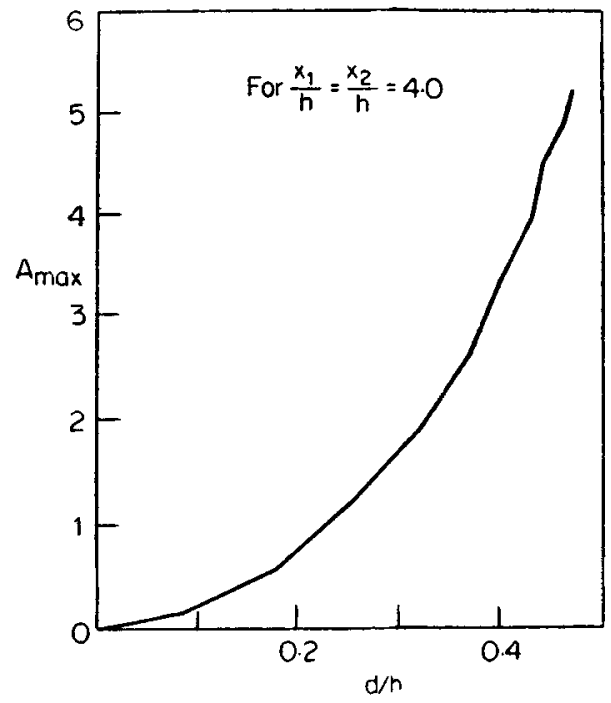

Figure 4. Plot of maximum anomaly produced by the structure at a point on its axis vs $d / h$.

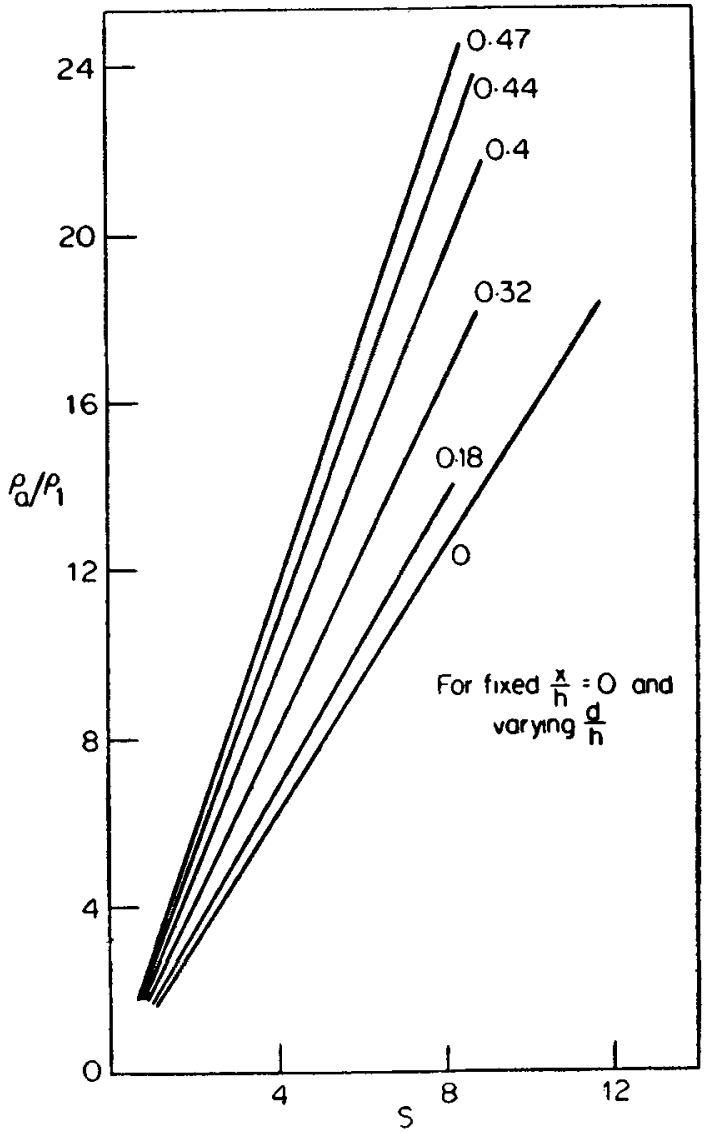

Figure 5. Plot of $\rho_{0} / \rho_{1}$ vs $S$ (conformal mapping technique). 


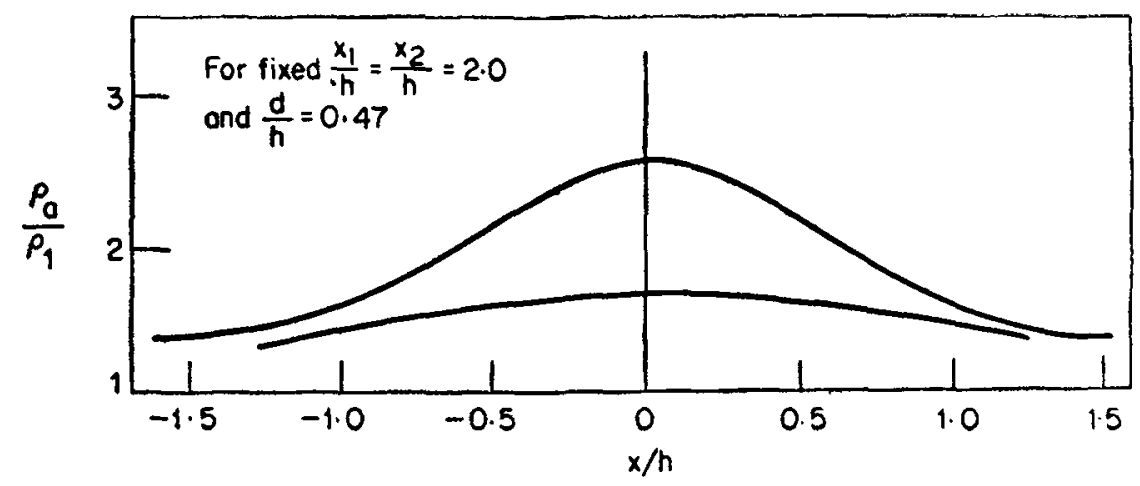

Figure 6. Plot of $\rho_{a} / \rho_{1}$ vs $x / h$ (FEM model II).

of the structure in order to solve it using a conformal mapping procedure (model I). Model II which is a progressive elaboration of model I has been solved here using the 'finite element method'.

The study has demonstrated (i) the potentiality of the finite element method in estimating resistivity anomalies over geological structures, not amenable to a closed form solution, which may be resorted to both for planning a resistivity survey as well as for interpretation of data. (ii) The anomaly produced by a buried hydrocarbon bearing structure of infinite resistivity can be detected with Ieasonable certainty if the ratio of its height to depth of its burial is of the order of $\mathbf{0 . 2}$. Further work is, however, needed to study the degree of uniqueness, obtainable in the solution of the corresponding inverse problem (Nek Ram 1976).

\section{Acknowledgements}

The authors are grateful to Prof. R S Mithal, former Head of the Department of Geology and Geophysics, University of Roorkee, Roorkee, for providing facilities to carry out this work, and to the Oil and Natural Gas Commission, Dehradun, for providing financial support. The authors are also thankful to Dr G C Nayak, Professor of Civil Engineering, University of Roorkee, for allowing the use of his computer program for finite element analysis. Thanks are also due to Prof. K N Khattri and Dr H Sinvhal, Department of Geology and Geophysics, University of Roorkee, Roorkee, for stimulating discussions.

\section{References}

Mufti I R 1976 Geophysics 4162

Nek Ram 1976 Direct detection of hydrocarbon through geo-electrical methods, M.Tech. Thesis, University of Roorkee, Roorkee (Unpublished)

Naidu P S 1967 Can. J. Earth Sci. 2351

Parasnis D S 1965 Geoexploration 32

Roy A and Jain S 1961 Geophysics 26229

Tranter C J 1956 Integral transforms in mathematical physics (New York : John Wiley) 